\title{
Análisis clínico-radiológico comparativo de tallos femorales no cementados planos de primera vs. segunda generación
}

\author{
Agustín O. Perea, Pablino Spesot, Matías García, Javier Arce, Sebastián García, \\ Ricardo Munafó Dauccia \\ Unidad de Cadera, Servicio de Ortopedia y Traumatología, Sanatorio de la Trinidad, \\ Ciudad Autónoma de Buenos Aires, Argentina
}

\begin{abstract}
RESUMEN
Introducción: El diseño del implante femoral es imprescindible para su adaptación a los distintos tipos de canales. El objetivo del estudio fue comparar la adaptabilidad del implante y el tipo de contacto de los tallos femorales planos de segunda generación. Materiales y Métodos: Se analizó a los pacientes sometidos a un reemplazo total de cadera bilateral en nuestra institución, entre 2007 y 2020, a quienes se les colocó un tallo de primera generación (Accolade TMZF) en una cadera y de segunda generación (Accolade II) en la contralateral. Se estudiaron las variables demográficas, la adaptación y el llenado del canal. Se analizó el Harris Hip Score modificado y el índice WOMAC. Resultados: Se incluyó a 42 pacientes (84 casos). En el grupo 1 (Accolade TMZF), la ocupación del canal fue del $81 \%$ y, en el grupo 2 (Accolade II), del 84\%. En el grupo 1, el contacto fue tipo 1 (60\%), tipo 2 (16\%) y tipo $3(24 \%)$. En el grupo 2, fue tipo $1(88 \%)$, tipo $2(7 \%)$ y tipo $3(5 \%)$. El Harris Hip Score modificado para el grupo 1 fue 88 y, para el grupo 2, 87,5. El puntaje WOMAC para el grupo 1 fue 2,5 y, para el grupo 2, de 3. Conclusión: La adaptabilidad del implante es fundamental para la estabilidad primaria y su osteointegración/fijación biológica. En nuestra muestra, es más precisa con tallos de segunda generación debido a las modificaciones del diseño.

Palabras clave: Tallos femorales planos; segunda generación; adaptabilidad femoral; fijación proximal mediolateral.

Nivel de Evidencia: IV
\end{abstract}

\section{Comparative Clinical-Radiological Analysis of First-Generation vs. Second-Generation Flat Cementless Femoral Stems}

\section{ABSTRACT}

Introduction: The design of the femoral implant is essential for its adaptation to the different types of femoral canals. The objective of this study is to describe the adaptability of the implant and the type of fixation (fill and fit) of second-generation flat femoral stems compared to first-generation femoral stems. Materials and Methods: We described the radiological characteristics of patients who had undergone bilateral total hip replacement between 2007 and 2020 in our institution with both a first-generation Accolade TMZF (Stryker Orthopedics) and a second-generation Accolade II (Stryker Orthopedics) flat cementless femoral implant. Demographic variables, adaptation, and canal filling were studied. The modified Harris Hip Score and the WOMAC index were analyzed. Results: Forty-two patients (84 cases) were included in the sample. In group 1 (Accolade TMZF) we obtained an average canal fill of $81 \%$ and in group 2 (Accolade II), we obtained an average of $84 \%$. In group 1, the type of fixation (fit) was type 1 (60\%), type $2(16 \%)$ and type $3(24 \%)$. In group 2, it was type $1(88 \%)$, type $2(7 \%)$ and type $3(5 \%)$. The modified Harris Hip Score for group 1 was 88 and, for group 2, 87.5. The WOMAC score for group 1 was 2.5 and for group 2 it was 3 . Conclusion: The adaptability of the implant is essential for primary stability and its osseointegration/biological fixation. This adaptability is more precise with second-generation flat femoral stems.

Keywords: Second-generation flat femoral stems; femoral adaptability; proximal mediolateral fixation.

Level of Evidence: IV Recibido 20-3-2021. Aceptado luego de la evaluación el 23-6-2021 • Dr. AGUSTín O. PEREA • agustinoscarperea@gmail.com ID https://orcid.org/0000-0002-7011-8966
Cómo citar este artículo: Perea AO, Spesot P, García M, Arce J, García S, Munafó Dauccia R. Análisis clínico-radiológico comparativo de tallos femorales no cementados planos de primera vs.
segunda generación. Rev Asoc Argent Ortop Traumatol 2021;86(4):475-482. https://doi.org/10.15417/issn.1852-7434.2021.86.4.1337 


\section{INTRODUCCIÓN}

El uso de tallos no cementados en el reemplazo articular de cadera se ha incrementado en los últimos 20 años debido a la gran demanda de pacientes cada vez más jóvenes y con mayores exigencias. La constante evolución de los distintos implantes hizo que su diseño geométrico, los materiales y el tipo de superficie se fueran modificando con el correr del tiempo. El diseño geométrico resulta ser fundamental para lograr una aceptable adaptación a los distintos tipos de canales femorales, ${ }^{1}$ con el objetivo de facilitar su osteointegración/fijación biológica y así lograr la máxima durabilidad del sistema.

Los implantes de segunda generación Accolade II (Stryker Orthopaedics, Mahwah, New Jersey, EE.UU.) tienen un diseño de cuña morfométrica con una curva medial variable, a fin de facilitar su adaptación a las diferentes geometrías del fémur proximal, más corto que el de primera generación y de anclaje metafisario medio lateral. Fueron diseñados con un programa especial (SOMA - Stryker, Orthopaedics), evaluando modelos en 3D con una base de datos de diferentes morfologías de canales femorales. Dicha base de datos recopila variantes de tamaño, forma y densidad óseos, así como edad, sexo y variaciones demográficas ${ }^{2,3}$ (Figura 1).
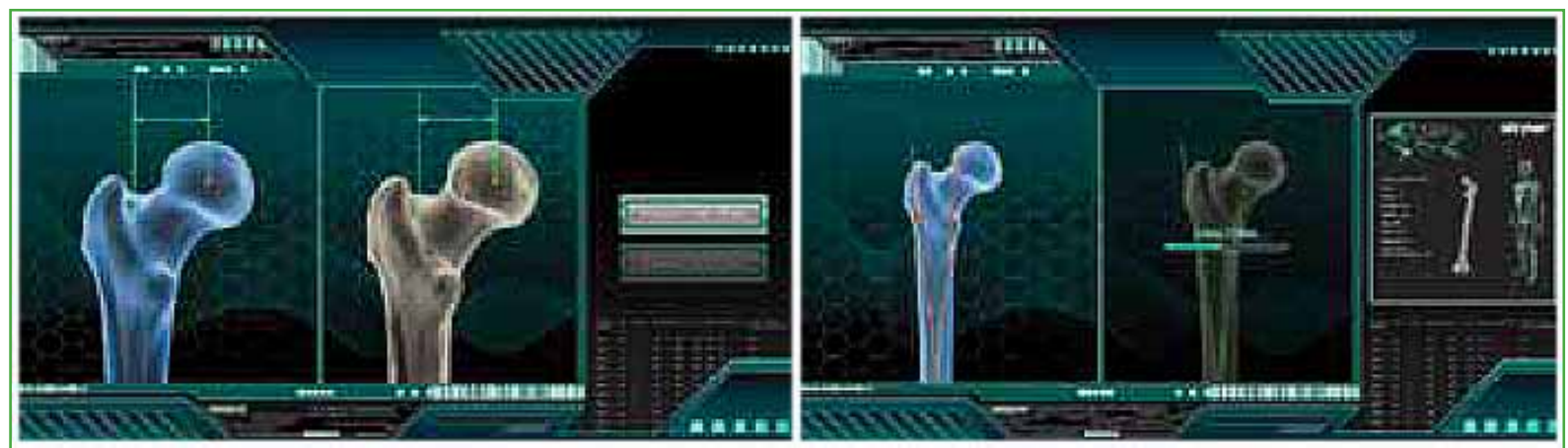

Figura 1. Modelos en 3D para el desarrollo del implante de segunda generación Accolade II.

El objetivo del estudio fue comparar la adaptabilidad y el tipo de contacto (adaptación y llenado del canal) entre los tallos femorales planos de segunda generación y los tallos femorales de primera generación respecto a la morfología del fémur proximal. La hipótesis de trabajo fue demostrar la versatilidad de los tallos femorales de segunda generación y su correlación con la evolución clínica basada en la morfología Dorr ${ }^{4}$ A y C.

\section{MATERIALES Y MÉTODOS}

Se llevó a cabo un estudio descriptivo, observacional, retrospectivo y longitudinal. Se recolectaron los datos de pacientes que habían sido sometidos a un reemplazo total de cadera bilateral entre 2007 y 2020.

En nuestra institución, se realizaron 3342 reemplazos articulares de cadera entre 2007 y 2020, el $89 \%$ fueron no cementados; el 9,2\%, híbridos y el 1,8\%, cementados. Se utilizó el tallo Accolade II en el 98,2\% de los reemplazos articulares no cementados. De dicha muestra, se incluyó a pacientes con reemplazo articular de cadera primario con un tallo femoral no cementado Accolade TMZF (Stryker Orthopaedics, Mahwah, New Jersey, EE.UU.) y, luego, en otra instancia, con un tallo femoral Accolade II (Stryker Orthopaedics, Mahwah, New Jersey, EE.UU.) en la cadera contralateral. Se excluyó a los pacientes con deformidades significativas metafiso-diafisarias (para ellos se eligió un implante con otras características de fijación).

Todos fueron operados por el mismo equipo quirúrgico, en la misma institución, bajo idéntico protocolo.

Los pacientes fueron divididos en dos grupos: grupo 1 (Accolade TMZF) y, luego de la cirugía de la cadera contralateral, en el grupo 2 (Accolade II). El seguimiento y el protocolo posoperatorio fueron los mismos para ambos grupos. 
Se realizaron controles radiológicos en el posoperatorio inmediato, a los meses 1, 3, 6 y 12, y luego anualmente. Se registraron las siguientes variables:

1. Edad en el momento de la cirugía.

2. Sexo.

3. Morfología del canal femoral según la clasificación de Dorr. ${ }^{4}$ Se analizó mediante radiografías y tomografía computarizada de ambas caderas.

4. Tipo de contacto, adaptación y porcentaje de llenado del canal del implante femoral en el plano coronal. Estas mediciones se hicieron en la radiografía de frente de ambas caderas al tercer mes de la cirugía, tomando como referencia la radiografía del posoperatorio inmediato, y estuvieron a cargo de dos de los autores. Se evaluó la confiabilidad interobservador e intraobservador de las mediciones de adaptación y llenado del canal seleccionando al azar 15 radiografías como en el estudio de Issa y cols. ${ }^{3}$ Se obtuvo un coeficiente de correlación de Pearson de 0,87 y 0,89 para la confiabilidad interobservador e intraobservador en las mediciones de adaptación y de 0,81 y 0,83 para la confiabilidad interobservador e intraobservador en las mediciones de ocupación del canal.

La adaptación del tallo femoral se evaluó en dos zonas: la región proximal (zona de recubrimiento de hidroxiapatita) y la región distal (zona desde la finalización del recubrimiento de hidroxiapatita hasta $10 \mathrm{~mm}$ proximal a la punta del tallo femoral). Se midió la brecha medial en la región proximal entre el tallo femoral y la cortical medial, y se obtuvo la distancia mínima (Pmin; mínima distancia desde la cortical medial hasta el implante) y máxima (Pmax; máxima distancia desde la cortical medial hasta el implante). En la región distal, se registró la brecha medial y lateral entre el tallo femoral y la cortical, y se obtuvo la distancia mínima (Dmin M; mínima distancia desde la cortical medial hasta el implante) y máxima (Dmax $M$; máxima distancia desde la cortical medial hasta el implante) medial y lateral ( $D \min L$; mínima distancia desde la cortical lateral hasta el implante y Dmax L; máxima distancia desde la cortical lateral hasta el implante) (Figura 2).
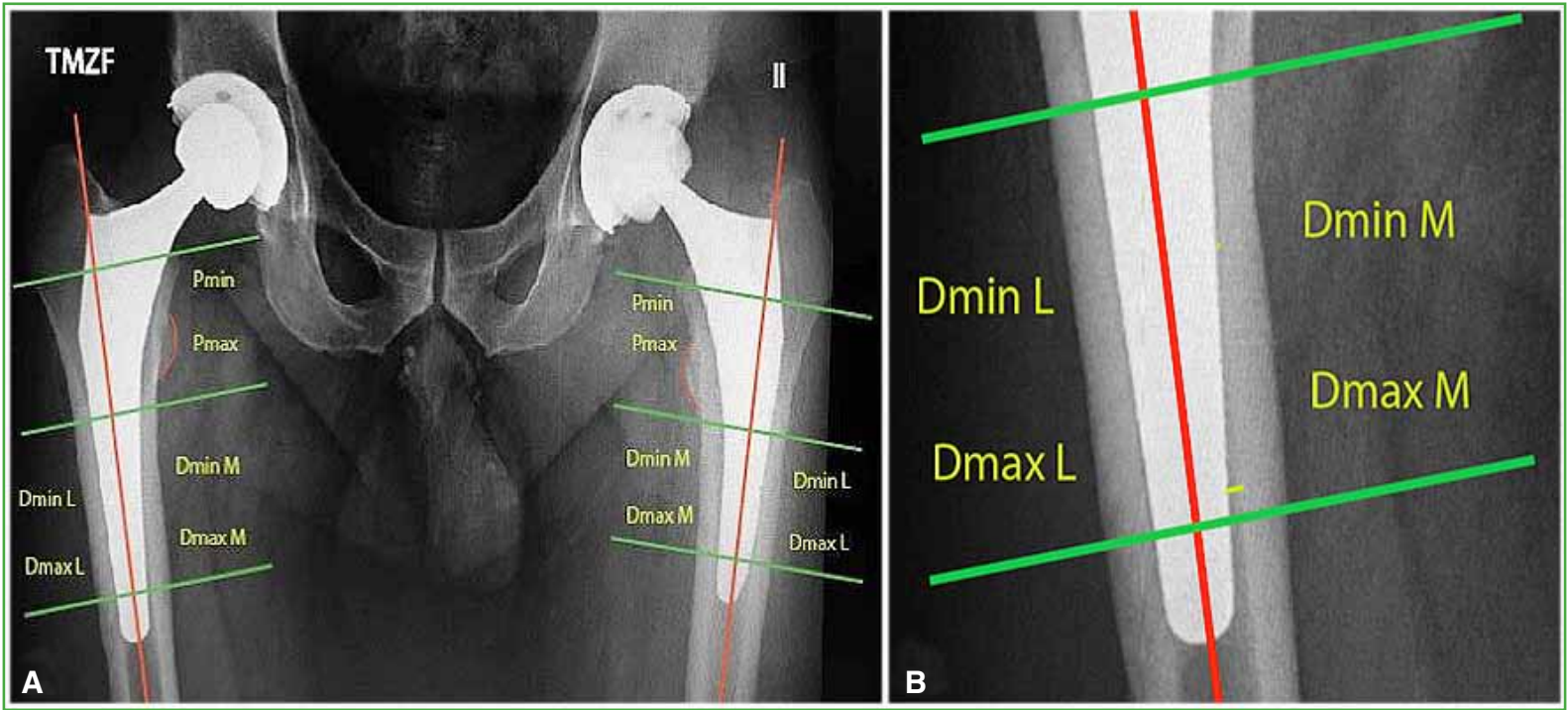

Figura 2. Adaptación del tallo femoral. A. Mediciones. B. Detalle. 
Con dichas medidas, se clasificó el contacto en: tipo 1 (global), cuando hay una fijación proximal y distal (la diferencia entre la brecha proximal y la distal es de $2 \mathrm{~mm}$ o menos), tipo 2 (proximal), cuando la fijación es solo proximal, y tipo 3 (distal) cuando el contacto es solo distal (Figura 3).

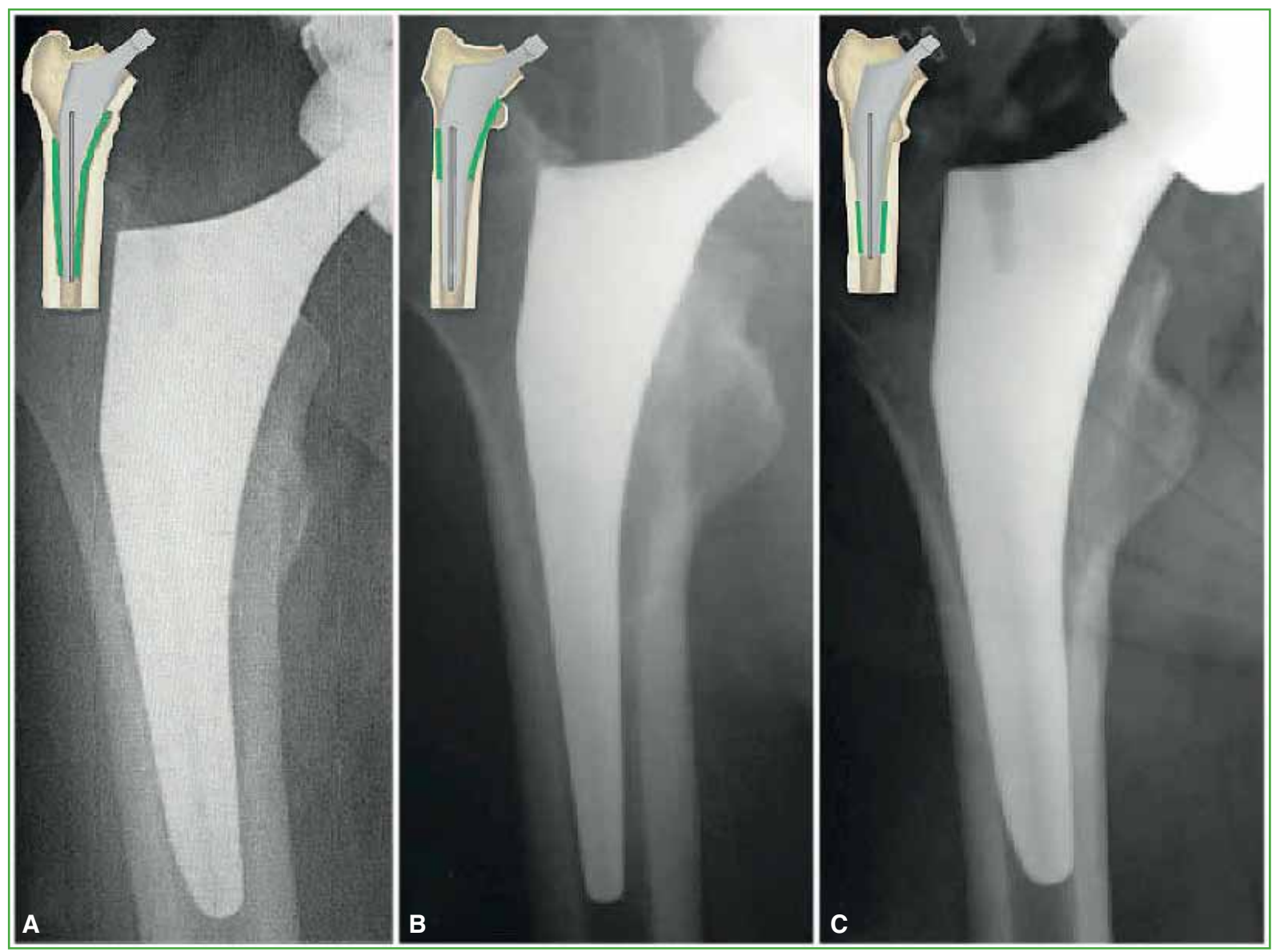

Figura 3. Tipos de contacto. A. Tipo 1 (global). B. Tipo 2 (proximal). C. Tipo 3 (distal).

El porcentaje de ocupación del canal femoral por parte del implante en el plano coronal (llenado del canal) se evaluó en tres zonas (medición del ancho del implante dividido el ancho del canal femoral): proximal a $10 \mathrm{~mm}$ por encima del trocánter menor (FP), medial a $60 \mathrm{~mm}$ por debajo del trocánter menor (F60) y distal a 2,5 mm proximal a la punta del tallo femoral (F2,5) (Figura 4).

5. Orientación del implante en valgo, varo y normal, analizando el eje del tallo femoral respecto al eje del canal femoral en el plano coronal.

6. Presencia de radiolucencias (radiolucidez alrededor del tallo femoral).

7. Hundimiento (migración caudal progresiva del implante $>1,5 \mathrm{~mm}$ ).

8. Aflojamiento (sintomatología asociada a imágenes radiológicas).

9. Infección posquirúrgica.

10. Fractura periprotésica intraquirúrgica o en el posoperatorio inmediato.

11. Luxación.

12. Harris Hip Score modificado (HHS modificado).

13. Western Ontario and McMaster (WOMAC). 


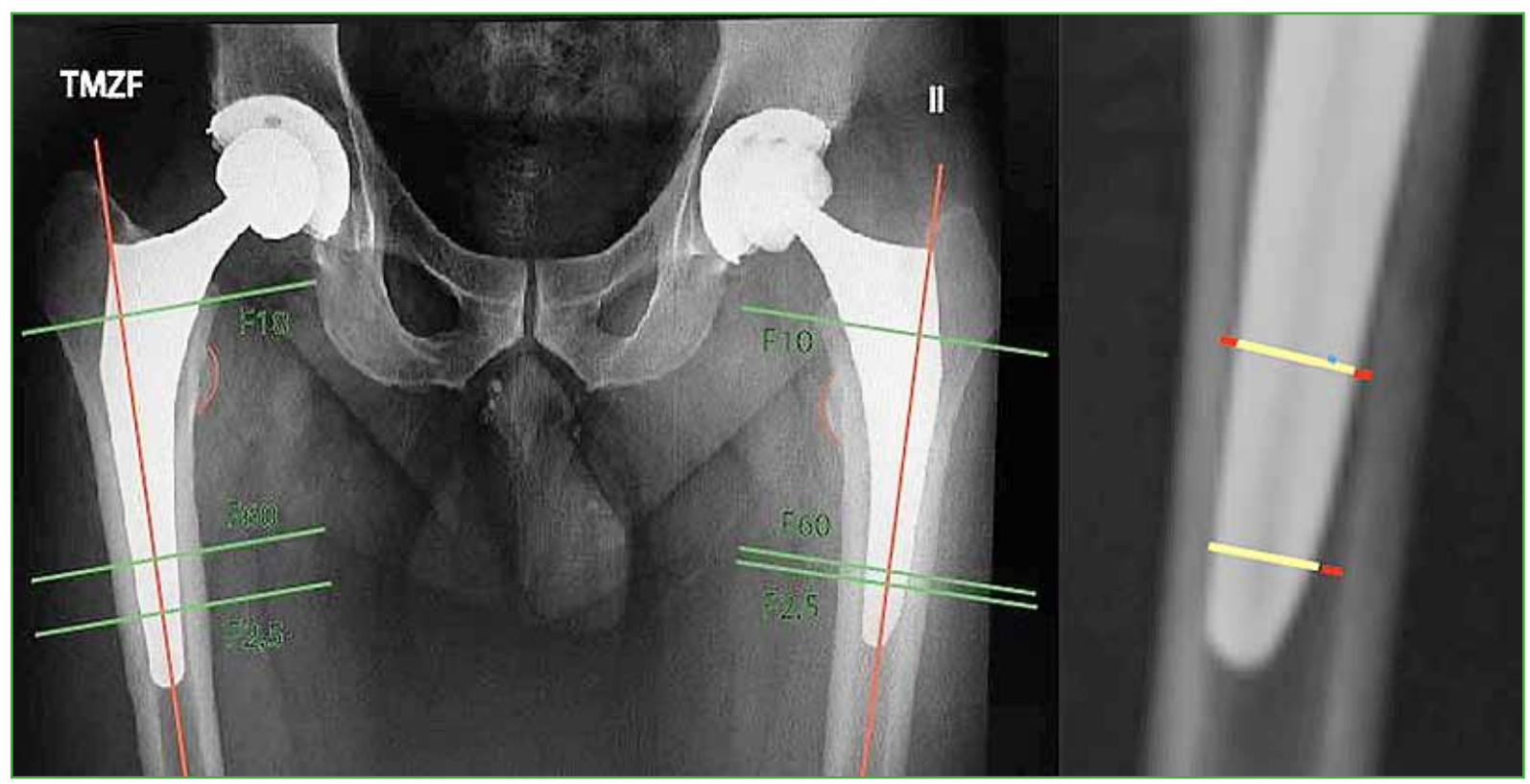

Figura 4. Ocupación del canal femoral (llenado del canal).

\section{Análisis estadístico}

Las variables categóricas se presentan con número absoluto y porcentaje; las variables continuas de distribución normal, con media y desviación estándar (DE); las de distribución asimétrica, con mediana, rango intercuartílico (Q1-Q3), mínimo y máximo. La distribución de las variables se evaluó mediante la prueba de Shapiro-Wilk. Se utilizó el estadístico adecuado para muestras apareadas según si la variable es continua o categórica, la distribución de los datos y el tamaño de muestra. El nivel de significancia estadística fue $\mathrm{p}<0,05$. Los datos fueron volcados en una planilla Excel y el programa estadístico utilizado fue RStudio.

\section{RESULTADOS}

Cuarenta y tres pacientes cumplieron con los criterios de inclusión. Uno fue eliminado por pérdida en el seguimiento; por lo tanto, la muestra quedó formada por 42 pacientes (84 casos). Veintidós $(52,4 \%)$ eran hombres. La media de la edad era de 59.3 años (DE 10 años) en el grupo 1 y de 65.1 años (DE 10 años) en el grupo 2, con una diferencia estadísticamente significativa $(\mathrm{p}<0,05)$. Los pacientes del grupo 1 habían sido operados entre 2007 y 2017, con un seguimiento hasta la fecha de 89.6 meses (rango 29-171); los del grupo 2, entre 2017 y 2020, con un seguimiento hasta la fecha de 27.7 meses (rango 8-41), con una diferencia estadísticamente significativa (p $<0,05$ ). El intervalo promedio entre ambas cirugías fue de 61.3 meses (rango 9-144). En los dos grupos, el implante acetabular utilizado fue Trident ${ }^{\circledR}$ Acetabular Cup (Stryker Orthopaedics, Mahwah, New Jersey, EE.UU.). El par de fricción de elección en ambos casos se adaptó a la edad y la demanda del paciente (Metal-Polietileno X3; Cerámica-Polietileno X3). Las características radiológicas principales se muestran en la Tabla 1.

Al comparar la adaptación y llenado del canal según la morfología femoral, en los canales Dorr A, el promedio de ocupación fue del $81 \%$ en el grupo 1 (Accolade TMZF) y del $86 \%$ en el grupo 2 (Accolade II). Estas cifras fueron del $79 \%$ y $86 \%$, respectivamente, para los Dorr B, y del 79\% y 77\% para los Dorr C, respectivamente. En cuanto a la adaptación en el grupo 1, en los canales Dorr A, predominaron el tipo de contacto 1 (50\%) y el tipo 3 (50\%) y no hubo tipo de contacto 2 en los cuatro casos de Dorr A. En los canales Dorr B, predominó el contacto tipo $1(64 \%)$, seguido del tipo $3(19 \%)$ y del tipo 2 (17\%). En los canales Dorr C, predominaron el tipo de contacto $2(50 \%)$ y el tipo $3(50 \%)$ y no hubo tipo 1 en los dos casos de Dorr C. Respecto a la adaptación en el grupo 2, en los canales Dorr A, predominó el contacto tipo $1(100 \%)$. En los canales Dorr B, predominó el contacto tipo 1 (89\%), y los tipos 2 y 3, ambos con 5,5\%. En los canales Dorr C, predominaron el tipo de contacto 1 (50\%) y el tipo $2(50 \%)$ y no hubo tipo 3 en los dos casos de Dorr C (Tabla 2). 
Tabla 1. Características radiológicas del grupo 1 Accolade TMZF y grupo 2 Accolade II.

\begin{tabular}{|c|c|c|c|}
\hline & $\begin{array}{c}\text { Grupo } 1 \\
\text { Accolade TMZF }\end{array}$ & $\begin{array}{c}\text { Grupo } 2 \\
\text { Accolade II }\end{array}$ & $\mathbf{p}$ \\
\hline $\begin{array}{l}\text { Dorr n }(\%) \\
\text { A } \\
\text { B } \\
\text { C }\end{array}$ & $\begin{array}{c}4(9) \\
36(86) \\
2(5)\end{array}$ & $\begin{array}{c}4(9) \\
36(86) \\
2(5)\end{array}$ & 0,9 \\
\hline $\begin{array}{l}\text { Adaptación* }(\mathrm{mm}) \mathrm{M}(\mathrm{Q} 1-\mathrm{Q} 2) \text { [mín-máx] } \\
\text { Brecha proximal } \\
\text { Brecha distal medial } \\
\text { Brecha distal lateral }\end{array}$ & $\begin{array}{c}2,5(2-3,5)[0,5-7] \\
1,5(0,5-2,37)[0-7] \\
1,5(0,5-2)[0-4]\end{array}$ & $\begin{array}{l}2,1(1,5-2,5)[0,5-4] \\
1,4(0,5-2)[0-6] \\
1,4(0,5-2)[0-4,5]\end{array}$ & $\begin{array}{c}0,0008 \\
0,339 \\
0,798\end{array}$ \\
\hline $\begin{array}{l}\text { Adaptación } \mathrm{n}(\%) \\
\text { Tipo } 1 \\
\text { Tipo } 2 \\
\text { Tipo } 3\end{array}$ & $\begin{array}{l}25(60) \\
7(16) \\
10(24)\end{array}$ & $\begin{array}{c}37(88) \\
3(7) \\
2(5)\end{array}$ & 0,009 \\
\hline $\begin{array}{l}\text { Llenado del canal** M (Q1-Q2) [mín-máx] } \\
\text { F10 } \\
\text { F60 } \\
\text { F2,5 }\end{array}$ & $\begin{array}{c}74(70,25-75,75)[58-85] \\
87(83-91,5)[57-100] \\
85,5(81,25-92)[58-100]\end{array}$ & $\begin{array}{c}75(72,25-80)[63-87] \\
90(85-94,75)[63-100] \\
90(85-94,75)[55-100]\end{array}$ & $\begin{array}{l}0,001 \\
0,013 \\
0,028\end{array}$ \\
\hline Llenado total del canal ${ }^{* * *} \mathrm{M}(\mathrm{Q} 1-\mathrm{Q} 2)$ [mín-máx] & $81(79-85,75)[64-93]$ & $84(82-89)[65-92]$ & 0,002 \\
\hline mHHS M (Q1-Q2) [mín-máx] & $88(84-91)[69-91]$ & $87,5(84-91)$ [59-91] & 0,4 \\
\hline WOMAC M (Q1-Q2) [mín-máx] & $2,5(0-6,5)[0-26]$ & $3(0-6)[0-38]$ & 0,8 \\
\hline
\end{tabular}

mHHS = Modified Harris Hip Score, WOMAC = Western Ontario and McMaster, $\mathrm{n}=$ valor absoluto, $\mathrm{M}=$ mediana, Q1 = primer cuartil, Q3 = tercer cuartil, mín = mínimo, máx = máximo. "Distancia en milímetros, ${ }^{* *}$ Porcentaje de ocupación del canal femoral, ${ }^{* * *}$ Porcentaje total de ocupación del canal femoral.

Tabla 2. Promedio del porcentaje según el tipo de contacto (adaptación) del tallo femoral y promedio del porcentaje de ocupación del canal femoral por parte del implante (llenado del canal) según la morfología del canal medular (Dorr)

\begin{tabular}{|l|c|c|}
\hline & Grupo 1 Accolade TMZF & Grupo 2 Accolade II \\
\hline Adaptación tipo 1/2/3 (\%) & & \\
Dorr A & $50 / 0 / 50$ & $100 / 0 / 0$ \\
\hline Dorr B & $64 / 17 / 19$ & $89 / 5,5 / 5,5$ \\
Dorr C & $0 / 50 / 50$ & $50 / 50 / 0$ \\
\hline Llenado del canal (\%) & & \\
Dorr A & 81 & 86 \\
Dorr B & 79 & 86 \\
Dorr C & 79 & 77 \\
\hline
\end{tabular}

En la Tabla 1, se detallan los puntajes del HHS modificado y WOMAC de ambas caderas en el posoperatorio alejado (7.4 años de promedio en Accolade TMZF y 2.3 años en Accolade II).

Todos los pacientes presentaron el mismo tipo de Dorr en ambos canales femorales.

La orientación de los tallos femorales fue en varo en tres casos $(7,14 \%)$ del grupo 1 y uno $(2,4 \%)$ del grupo 2. La orientación fue en valgo en dos $(4,8 \%)$ tallos del grupo 1 y uno $(2,4 \%)$ del grupo 2 . Los restantes 77 (91,7\%) tallos femorales tuvieron una orientación normal.

No se observaron radiolucencias, hundimiento del implante, ni aflojamientos en ninguno de los 84 (100\%) reemplazos de cadera a lo largo de la evaluación. Un paciente (grupo 2) sufrió un proceso infeccioso que se resolvió con desbridamiento quirúrgico y recambio de componentes móviles. Uno del grupo 1 tuvo una fractura de trocánter mayor a los tres años de la cirugía por caída de propia altura, con tratamiento incruento. 
Se registraron dos episodios de luxación en un mismo paciente, dentro de los tres meses de la operación, en la primera cirugía (Accolade TMZF) luxación anterior y la segunda cirugía (Accolade II) luxación posterior, resueltas con reducción cerrada, sin otro episodio hasta la fecha.

\section{DISCUSIÓN}

Se deben considerar diferentes aspectos al elegir el implante ideal: el material de fabricación, la superficie, el modo de fijación y su geometría. La evolución de los diferentes diseños de componentes femorales apunta a mejorar los resultados y la satisfacción de los pacientes.

El diseño del Accolade TMZF de primera generación tiene su origen en el tallo autobloqueante de Muller ME, trapezoidal plano, que fue modificado conservando, en parte, esas características para que pueda adaptarse a las necesidades actuales de la cirugía de reconstrucción de cadera.

Una aleación de titanio, molibdeno, zirconio y hierro en su diseño actual incrementa su módulo de elasticidad para hacerla más cercana al del hueso, y mejora sensiblemente las transferencias de cargas respecto a otros materiales. La superficie del Accolade tratada con "Pure Fix HA" (hidroxiapatita) acelera el proceso de fijación biológica, esto asociado a su diseño produce una excelente combinación para lograr la estabilidad inicial y la integración, lo que mejora la durabilidad del sistema.

Sin embargo, se encontraron algunos inconvenientes ligados a su adaptabilidad a los diferentes tipos geométricos del fémur proximal, especialmente Dorr A y C. 5

El nuevo diseño Accolade II fabricado con los mismos materiales y la misma superficie tiene modificaciones en su diseño geométrico que permiten sortear los inconvenientes de adaptabilidad que se plantearon con el diseño TMZF.

Issa y cols. analizaron parámetros de adaptación de los tallos femorales Accolade TMZF y Accolade II en 100 reemplazos totales de cadera, y hallaron un porcentaje elevado de mejor llenado del canal en el tallo de segunda generación (Accolade II) 90,6\% vs. 85,3\% a nivel medio y $88,1 \%$ vs. $76,6 \%$ a nivel distal, respecto del tallo de primera generación (Accolade TMZF), ${ }^{3}$ probablemente debido a que la curva medial en el Accolade TMZF es fija y, en el Accolade II, es variable, una de sus principales modificaciones.

Se han publicado artículos sobre la adaptación del tallo femoral y sus complicaciones, como ausencia de osteointegración, hundimiento, fracturas periprotésicas intraoperatorias o en el posoperatorio inmediato, dolor residual de muslo, etc. ${ }^{5-8}$ La geometría del implante, el material de fabricación, la edad y la calidad ósea del huésped, la técnica de colocación y la relación del diseño del implante-fémur son factores señalados en estos reportes. ${ }^{1,9,10} \mathrm{~A}$ fin de disminuir el micromovimiento y facilitar la osteointegración, es necesario el contacto íntimo entre el hueso cortical y el implante. ${ }^{11}$ En nuestro estudio, observamos un predominio en el tipo de contacto global (tipo 1) en el grupo 2 del 88\% comparado con el 60\% en el grupo 1.

En cuanto a la adaptación del implante a los diferentes tipos de canales femorales, creemos que la variabilidad de la curva medial permite una mejor adaptación a los fémures de los tipos A y B (según Dorr) y, de esta manera, un mayor contacto medial para facilitar la transmisión de cargas del implante a la cortical femoral medial y un adecuado contacto metafiso-diafisario permitiendo el correcto funcionamiento de la superficie porosa de integración.

No hubo diferencias significativas en la evaluación clínico-funcional de ambos grupos en los puntajes HHS modificado y WOMAC (p 0,4 y 0,8), respectivamente. El 95,2\% (40 pacientes) del grupo 1 superó 74 puntos en el HHS modificado, considerada una mejoría aceptable ${ }^{12}$ y el 92,8\% del grupo 2 (3 pacientes), es decir, un buen resultado funcional general en ambos grupos.

Como limitaciones del estudio podemos mencionar la cantidad de pacientes $(n=42)$, principalmente para analizar la adaptabilidad del implante en los tipos morfológicos de fémur menos frecuentes (Dorr A y C) y que, en general, están asociados a inconvenientes en la implantación. El seguimiento del grupo 2 es corto (de 8 a 41 meses, promedio 27.7 meses) para evaluar los criterios radiológicos a largo plazo del implante o las complicaciones relacionadas con el implante. La evaluación clínica de ambos grupos fue con una evolución diferente (promedio 7.4 vs. 2.3 años) sin contar con un registro clínico preoperatorio, además de una diferencia etaria promedio de ambos grupos de 5.8 años (promedio de edad Accolade TMZF 59.3 años y Accolade II 65.1 años). Las mediciones radiológicas se hicieron en el plano coronal (por ser un implante de fijación medio-lateral), no se realizaron mediciones en el plano sagital; por lo tanto, el porcentaje de ocupación del canal es relativo (por lo general, un llenado deficitario en la radiografía de perfil, ya que no es el modo de fijación de los tallos femorales planos). 


\section{CONCLUSIONES}

La adaptabilidad del implante en el canal femoral es fundamental para facilitar su osteointegración/fijación biológica y, en nuestra muestra, es más precisa con tallos femorales planos de segunda generación. Creemos que su uso es recomendable en los canales Dorr A y B, y tiene una muy buena adaptabilidad en los Dorr C. Como ya se mencionó, es necesario contar con una muestra más grande, principalmente para analizar su adaptabilidad en los canales Dorr A y C. También es indispensable un seguimiento más extenso en el grupo 2, porque los resultados locales y mundiales con el Accolade TMZF) son excelentes en relación con la fijación, integración y duración del implante.

Conflicto de intereses: Los autores no declaran conflictos de intereses.

ORCID de P. Spesot: https://orcid.org/0000-0001-7281-4212 ORCID de M. García: https://orcid.org/0000-0001-7820-3578 ORCID de J. Arce: https://orcid.org/0000-0002-9084-7891
ORCID de S. García: https://orcid.org/0000-0003-2630-6350 ORCID de R. Munafó Dauccia: https://orcid.org/0000-0003-0300-7841

\section{BIBLIOGRAFÍA}

1. Pierce TP, Jauregui JJ, Kapadia BH, Elmallah RK, Cherian JJ, Harwin SF, et al. Second-generation versus firstgeneration cementless tapered wedge femoral stems. Orthopedics 2015;38(9):550-4. https://doi.org/10.3928/01477447-20150902-04

2. Wuestemann T, Bastian A, Schmidt W, Cedermark C, Streicher R, Parvizi J, et al. A novel technique for studying proximal femoral bone morphology for hip implant design. EFORT - European Federation of National Associations of Orthopaedics and Traumatology (11th Congress); 2011.

3. Issa K, Pivec R, Wuestemann T, Tatevossian T, Nevelos J, Mont MM. Radiographic fit and fill analysis of a new second-generation proximally coated cementless stem compared to its predicate design. J Arthroplasty 2014;29(1):192-8. https://doi.org/10.1016/j.arth.2013.04.029

4. Dorr LD, Faugere MC, Mackel AM, Gruen TA, Bognar B, Malluche HH. Structural and cellular assessment of bone quality of proximal femur. Bone 1993;14(3):231-42. https://doi.org/10.1016/8756-3282(93)90146-2

5. White CA, Carsen S, Rasuli K, Feibel RJ, Kim PR, Beaulé PE. High incidence of migration with poor initial fixation of the Accolade1 stem. Clin Orthop Relat Res 2012;470(2):410-7. https://doi.org/10.1007/s11999-011-2160-Z

6. Brown TE, Larson B, Shen F, Moskal JT. Thigh pain after cementless total hip arthroplasty: evaluation and management. J Am Acad Orthop Surg 2002;10(6):385-92. https://doi.org/10.5435/00124635-200211000-00002

7. Colacchio ND, Robbins CE, Aghazadeh MS, Talmo CT, Bono JV. Total hip intraoperative femur fracture: do the design enhancements of a second-generation tapered-wedge stem reduce the incidence? J Arthroplasty 2017;32(10):3163e3168. https://doi.org/10.1016/j.arth.2017.05.012

8. Fleischman AN, Schubert MM, Restrepo C, Chen AF, Rothman RH. Reduced incidence of intraoperative femur fracture with a second-generation tapered wedge stem. J Arthroplasty 2017;32(11):3457e3461. https://doi.org/10.1016/j.arth.2017.06.018

9. Engh CA, Massin, P, Suthers KE. Roentgenographic assessment of the biologic fixation of porous-surfaced femoral components. Clin Orthop Relat Res 1990;(257):107-28. PMID: 2199114

10. Nam D, Salih R, Barrack R, Ryan M Nunley RN. An evaluation of proximal femur bone density in young, active patients undergoing total hip arthroplasty at one year postoperatively. Hip Int 2019;29(1):51-7. https://doi.org/10.1177/1120700018761152

11. Bochatey EJ, Lopreite F. Resultados a corto plazo de la artroplastia total de cadera con tallos femorales de fijación metafisaria de segunda generación. Rev Asoc Argent Ortop Traumatol 2020;85(3):214-21. https://doi.org/10.15417/issn.1852-7434.2020.85.3.1021

12. Kvien TK, Heiberg T, Hagen KB. Minimal clinically important improvement/difference (MCII/ MCID) and patient acceptable symptom state (PASS): what do these concepts mean? Ann Rheum Dis 2007;66(Suppl 3):iii40-1. https://doi.org/10.1136/ard.2007.079798 